\title{
Relation between Cytokines and Brucella Arthritis, Spondylitis, and Sacroiliitis
}

\author{
Nayyereh Saadati ${ }^{1}$, Mandana Khodashahi ${ }^{{ }^{*}}$, Bahram Naghibzadeh ${ }^{2}$, Rozita Khodashahi ${ }^{3}$
}

\author{
${ }^{1}$ Rheumatic Diseases Research Center, Mashhad University of Medical Sciences, Mashhad, Iran. ${ }^{2}$ Mount Medical \\ Center, Australia. ${ }^{3}$ Department of Infectious Diseases, School of Medicine, Mashhad University of Medical Sciences, \\ Mashhad, Iran.
}

\begin{abstract}
Cytokines are produced and secreted from T cells and other various cells. Cytokines play an effective role in the responses elicited by the immune system. As brucella infection is caused by an intracellular aerobic rod, cellular immunity has a considerable effect on this disease. The aim of the present study was to evaluate the relation between cytokines and brucellosis arthritis, spondylitis, and sacroiliitis.

This descriptive case-control study was conducted in two tertiary hospitals in Mashhad, Iran in 2010. The study population consisted of a case group comprising patients diagnosed with brucella arthritis with lower back pain and a control group comprising normal healthy participants. The diagnosis of brucellosis was investigated through history-taking, physical examinations, and serologic examinations. The interferon gamma (IFN $\gamma$ ), interleukin 2 (IL-2), interleukin 4 (IL-4), interleukin 10 (IL-10), and tumor necrosis factor alpha $(\mathrm{TNF} \alpha)$ in the serum samples of both groups were measured using an enzyme-linked immunosorbent assay technique.

The IL-10 was significantly different in the brucella and control groups $(P$ value $=0.02)$. However, IL-2, IL-4, and TNF $\alpha$ were comparable between the brucella and control groups $(P$ value $=0.1)$. A significant difference was observed between IFN $\gamma$ and brucella in the control group $(P$ value $=0.05)$.

IFN $\gamma$ and IL-10 levels were higher in the brucella group than in the control group.
\end{abstract}

Keywords: Cytokines; Brucella; Arthritis; Spondylitis; Sacroiliitis

\section{Introduction}

Brucellosis, a prevalent infectious disease around the world, is caused by intracellular bacteria belonging to the genus Brucella with tropism for the reticuloendothelial system [1]. Brucella species are gram-negative facultative intracellular bacteria that can cause serious diseases in animals and humans [2-4]. Peripheral arthritis, sacroiliitis, and spondylitis are three distinct forms of osteoarticular disease, which is the most common complication of brucellosis [1].

There is evidence in the literature of the antibody production against brucella in the human body, but. because brucella bacteria are intracellular, cell-mediated immunity is the final mechanism to kill these bacteria. Cellular immunity studies mostly performed on animal models have noted that tumor necrosis factor alpha (TNF $\alpha)$ and interleukin 12 (IL-12) are significant in the defense mechanism against Brucella abortus [5].

The secretion of cytokines, such as interleukin 12 (IL-12), interferon gamma (IFN $\gamma$ ), tumor TNF $\alpha$, and others, plays an important role in intracellular destruction and phagocyte apoptosis; however, the potential effects of cytokine blocking in vivo and, in particular, TNF $\alpha$ blocking in the course of human brucellosis are not clear. IFN $\gamma$ levels increase in the presence of brucella infection. If a patient cannot produce a sufficient amount of IFN $\gamma$, the infection will remain in the body. Based on the literature, increased levels of IFN $\gamma$ as well as interleukin 10 (IL-10) have been demonstrated in brucellosis compared to normal individuals [4].

Moreover, the role of the cytokine network in the pathogenesis of spondylitis and sacroiliitis has been discussed in recent years $[6,7]$. Patients with spondylitis have been reported to have increased plasma levels of soluble tumor

Personal non-commercial use only.Rheumatology Research Journal. Copyright (C) 2019. All rights reserved

*Corresponding Author: Mandana Khodashahi, MD, Assistant professor of Rheumatology, Rheumatic Diseases Research Center, Mashhad University of Medical Sciences, Mashhad, Iran, Email: khodashahimn@mums.ac.ir, Tel/Fax: (+98) 513-8012753, (+98) 513-8401036 Po Box: 9176699199

Received: 02 May 2018 ; Accepted: 16 January 2019 
necrosis factor receptor 1, soluble tumor necrosis factor receptor 2, and osteoprotegerin compared with the general population [8]. The symptoms of spondylitis and sacroiliitis are not usually observable. Furthermore, symptoms such as difficulty walking and pressure on the spinal cord may occur in severe cases or cases with extensive involvement. The most common area of involvement is the lumbar spine $[9,10]$.

Sacroiliitis after brucellosis, especially in adults, is mainly dominant skeletal brucellosis. It is reported more in the Mediterranean and the Middle East than in other areas. This may be due to the high prevalence of Brucella melitensis in these areas [2].

In general, sacroiliitis affects the patient with severe pain and movement limitation in its acute stage [11]. Since brucellosis generates significant costs in animal production and public health [2-4], further studies on the factors affecting these diseases are very important. According to the high prevalence of brucellosis, it remains a difficult problem to be tackled in endemic areas, such as Iran. The present study evaluated the relations between cytokines and brucellosis arthritis, spondylitis, and sacroiliitis.

\section{Materials and Methods}

This descriptive case-control study was performed on healthy cases in a control group and patients with brucella arthritis and back pain, spondylitis, and sacroiliitis in a brucella group admitted to two main tertiary hospitals in Mashhad in 2010.

\section{Inclusion and exclusion criteria}

Pregnancy and smoking were the exclusion criteria. None of the participants had received anti-inflammatory drugs, except acetaminophen, such as corticosteroids and other antibiotics, before entering the study.

\section{Study design}

The participants were divided into two groups. The control group consisted of healthy volunteer blood donors $(n=15)$ and medical students $(n=25)$. The intervention group consisted of 40 patients diagnosed with brucella arthritis, back pain, spondylitis, and sacroiliitis. Each participant completed a questionnaire collecting demographic information. Data collection lasted for 18 months. The standard sero-agglutination test with a titer of $\geq 1.320$ and the 2-mercaptoethanol brucella agglutination test with a titer of $\geq 1.40$ with clinical symptoms were the diagnostic criteria for brucellosis. Samples of $10 \mathrm{cc}$ of blood were collected from the patients for the enzyme-linked immunosorbent assay (ELISA) and sent to the immunology section of the Ghaem Hospital laboratory.

After the centrifugation and separation of the serum, each sample was kept at $-70^{\circ} \mathrm{C}$ (Giant Star, Bixolon Company) for 18 months. The number of cytokines was measured using the ELISA method. Firstly, a 100- $\mu$ l sample of the specific antibody of each cytokine produced in the Mani Teb Company was pipetted in a 96-cell microplate. After $16 \mathrm{~h}$ of incubation, it was washed three to four times using PBS-Tween in a refrigerator temperature of $2-4{ }^{\circ} \mathrm{C}$; after blocking the free space of the microplate with $1 \%$ bovine serum albumin, a diluted sample was added to each cell and incubated for $30 \mathrm{~min}$ at $37^{\circ} \mathrm{C}$. After washing, a second layer antibody, which was conjugated with peroxidase radish-horse enzyme, was added to each cell as a label. After 30 min of incubation, the substrate (H2O2) and chromogen were added, and after 10-15 min, the stop solution was added. Then, the concentration of each sample was obtained using standard samples at a wavelength of $450 \mathrm{~nm}$.

The antibody levels were assayed using the Brucella Wright, agglutination, and 2-mercaptoethanol tests. Then the cytokines IL-2, IL-4, and IL-10 were measured using kits. IFN $\gamma$ and TNF $\alpha$ were measured by the R\&D System (Canada) using ELISA technique. Magnetic resonance imaging was utilized to determine sacroiliitis. No intervention was conducted regarding the treatment of the patients. The treatment protocols were performed based on the recommendation of the World Health Organization guidelines [2].

\section{Statistical analysis}

The data was analyzed in SPSS software (version 22) using the t-test and the Kruskal-Wallis test. A $P$ value $<0.05$ was considered statistically significant.

\section{Ethical Considerations}

Informed consent was obtained from all participants. To observe the ethical considerations, all cases were informed about the purpose of the study. Furthermore, participants were assured about the possibility of withdrawing from the study at any stage of the project.

\section{Results}

There were 23 male and 17 female cases with a mean age of $39.6 \pm 23.2$ years. Brucellosis was diagnosed based on physical examinations and serologic tests. The results of the Kolmogorov-Smirnov test showed the normal distribution of IL- 2 and IL-10 levels. The IL-4, IFN $\gamma$, and TNF $\alpha$ levels had non-normal distributions. The IFN $\gamma$ and IL-10 levels were significantly higher in the brucella group compared with those in the case group. The results of the t-test showed a significant difference in IL-10 levels between the two groups $(P$ value $<0.001)$. However, the IL-2 level was not different between the two groups $(P$ value $=0.1)$.

The Kruskal-Wallis test revealed a significant difference with respect to IFN $\gamma$ between the two groups $(P$ value $=0.05)$. However, the TNF $\alpha$ and IL-4 levels were not significantly different between the groups $(P$ value $=0.1)$. These results reflected a cell-mediated immunity response involving $\mathrm{T}$ helper cells. The Wright test 
titer was $1 / 160-1 / 1280$ in $90 \%$ of the brucella group, and the 2-mercaptoethanol test titer was $1 / 80-1 / 320$ in $90 \%$ of the cases. Table 1 tabulates the measurement of cytokines IL-2, IL-4, IL-10, TNF $\alpha$, and IFN $\gamma$ among the investigated samples. The results revealed a significant difference re- garding IFN $\gamma$ between the two groups $(P$ value $=0.05)$. The t-test results showed a significant difference regarding IL10 between the two groups $(P$ value $=0.01)$. The findings indicated that IFN $\gamma$ and IL-10 played important roles in the pathogenesis of brucella arthritis and back pain.

Table 1. Comparison of (mean $\pm \mathrm{SD}$ ) serum cytokines levels $(\mathrm{pg} / \mathrm{mL})$ in brucella and control groups

\begin{tabular}{lccc}
\hline \multicolumn{1}{c}{ Cytokine } & Brucella group & Control group & P value \\
\hline Tumor necrosis factor alpha (mean $\pm \mathrm{SD})$ & $1069.5( \pm 270.36)$ & $862.11( \pm 667.65)$ & 0.1 \\
Interleukin 2 (mean $\pm \mathrm{SD})$ & $544.87( \pm 334.03)$ & $254.56( \pm 281.02)$ & 0.1 \\
Interferon gamma $($ mean $\pm \mathrm{SD})$ & $243.32( \pm 49.72)$ & $155.73( \pm 119.44)$ & 0.05 \\
Interleukin 4 (mean $\pm \mathrm{SD})$ & $26.04( \pm 42.41)$ & $7.21( \pm 8.46)$ & 0.1 \\
Interleukin 10 $($ mean $\pm \mathrm{SD})$ & $522.97( \pm 154.72)$ & $119.12( \pm 69.29)$ & 0.001 \\
\hline
\end{tabular}

\section{Discussion}

The results of this study showed increased levels of IFN $\gamma$ and IL-10 in the brucella group compared with the control group. These cytokines are predominantly secreted from type 1 helper $\mathrm{T}$ cells, which play an important role in cell-mediated immunity. In addition to type 1 helper $\mathrm{T}$ cells, activated antigen-presenting cells affect cell-mediated immunity against brucella [12]. Efforts have been made to induce cell-mediated immune responses in the development of a vaccine against brucella [13]. Cytokines are soluble mediators secreted from lymphocytes, in particular helper T cells, after antigen stimulation and affect cells in a similar way as macrophages and natural killer (NK) cells in the modulation of immune responses [14].

IL-12 is mostly secreted by macrophages in response to intracellular pathogens and is an important factor for $\mathrm{T}$ lymphocytes and NK cells in the production of IFN $\gamma[3$, 4]. The evidence has shown the role of IL-12 in resistance against brucella by the stimulation of IFN $\gamma$ production and the enhancement of NK cell activity [5]. Increased levels of IL-2 in human brucellosis have been reported. However, IL-2 and IL-4 levels were not significantly different between the two groups. According to the literature, IL-2 was not found in brucella patients [4]. Again in another study, the presence of IL-2 in brucella was not reported [15].

Another study reported that the spleen of rat models experimentally infected with brucella had low levels of IL-2 production. IFN $\gamma$ is a cytokine that is secreted not only by type 1 helper T cells, but also by NK cells and CD8-positive lymphocytes. This cytokine activates macrophages to kill intracellular bacteria. Moreover, it activates macrophages and NK cells and plays an important role in immunity against intracellular pathogens [16].

In the present study, IFN $\gamma$ had the highest level in bru- cella patients compared to the assayed cytokines of other cases. High IFN $\gamma$ levels in brucella have been previously reported [4]. Regarding IL-4, a slightly significant difference existed between the cases with brucella and healthy subjects. According to the literature, high levels of IL-4 were reported $[17,18]$. Likewise, IL-2 levels had no significant difference between brucella and healthy patients. No studies were observed regarding the IL-2 in human brucella; however, some studies in animal models have reported high levels of IL-10 in the spleen of rats with brucella. The results of another study revealed the down-regulation of immune response by the presence of this cytokine in rats [19].

In the present study, high levels of IFN $\gamma$ and IL-10 were observed in brucella patients. These cytokines are secreted mainly by type 1 helper T cells. Cytokines secreted by type 1 helper T cells direct cell-mediated immunity towards an effective defense against intracellular pathogens and are probably effective in the improvement of such infections [16]. In addition to type 1 helper $T$ cells activation, regulatory $\mathrm{T}$ cells have gained attention, especially when brucellosis becomes chronic. It has been shown that the IL-2 receptor alpha chain+forkhead box $\mathrm{P} 3+$ regulatory $\mathrm{T}$ cells have a lower percentage in chronic brucellosis compared to other cells [20]. In some limited studies, IL-1 and IL-4 levels were not detected in brucella patients [21]. A previous study reported low levels of TNF $\alpha$ [22]. Similarly, TNF $\alpha$ was not observed in the present study.

In a recent study, the role of toll-like receptors (TLR) was demonstrated to affect the production of IL- 6 and IL-12 simulated by Brucella RNA in vitro. The authors added that brucella ribonucleic acid had significant immunostimulatory function, and the cytokines released by dendritic cells in the response to this stimulation were TLR-dependent [23]. In another study carried out on 173 patients with 
brucellosis and 75 healthy controls, the IL-2 gene polymorphisms (positions +114/-384) brought the authors to the conclusion that the inheritance of TT genotype (position +114) and TT/TT haplogenotype (+114/-384) were higher in the control group compared to those in the case group and can be responsible for the resistance to brucellosis [24].

The results of the present study revealed that the type 1 helper $\mathrm{T}$ cell response was more important than the type 2 helper $\mathrm{T}$ cell response in brucella infection; in addition, the continuation of this response was important in the progression of the infection. According to the findings of this study, there was a significant difference in L-10 between the two groups. However, there was no significant difference in IL-2, IL-4, and TNF $\alpha$ between the two groups. The results of the Kruskal-Wallis test showed a significant difference in IFN $\gamma$ between the two groups. The IL-10 and IFN $\gamma$ levels were significantly higher in the brucella group than in the control group.

In this study, the cytokines produced in patients with brucella arthritis and back pain were evaluated. However, the patients with peripheral or axial arthritis were not separated. Further studies are suggested to investigate the relation between cytokines and brucella arthritis based on peripheral or axial arthritis. One limitation of this study was the unmatched case and control groups. Other limi- tations were the small sample size and the lack of a control group without joint involvement. Although the subject of this study was previously investigated in patients with brucellosis in Azarbaijan province, Iran, this re-evaluation was performed to confirm the previous findings.

\section{Conclusion}

IFN $\gamma$ and IL-10 had confirmatory roles in brucella patients suffering from brucella arthritis and back pain. This response is not related to the severity of the infection. IFN $\gamma$ was responsible for type 1 helper $\mathrm{T}$ cell response in brucella arthritis and back pain. IFN $\gamma$ and IL-10 levels were significantly higher in the brucella group than the control group. Due to the importance of the present subject, it is recommended that studies be performed with a larger sample size and which cover wider ranges both in cases and controls. The authors plan to investigate the relation between serum zinc and copper levels in patients with brucella in the next project.

\section{Acknowledgments}

This project was approved by the Ethics Committee of Mashhad University of Medical Sciences. The authors would like to thank Mrs. Farzaneh Iravani and Ms. Azam Brook for their genuine cooperation.

\section{Conflict of Interest}

The authors declare no conflicts of interest. 


\section{References}

1. Aktar F, Tekin R, Bektaş MS, Güneş A, Köşker M, Ertuğrul S. et al. Diagnostic role of inflammatory markers in pediatric Brucella arthritis. Ital J Pediatr 2016; 42(1):3. doi: 10.1186/s13052-016-0211-5.

2. Mirnejad R, Jazi FM, Mostafaei S, Sedighi M. Epidemiology of brucellosis in Iran: A comprehensive systematic review and meta-analysis study. Microb Pathog 2017; 109:239-47. doi: 10.1016/j.micpath.2017.06.005.

3. Bosilkovski M, Dimzova M, Grozdanovski K. Natural history of brucellosis in an endemic region in different time periods. Acta Clin Croat 2009; 48(1):41-6.

4. Dashti AS, Karimi A, Elyasi B, Shamshiri AR, Abdolmajid S. Cytokine profiles in acute brucellosis, compared with those in other febrile illnesses. Arch Clin Infect Dis 2017; 12(4). doi: 10.5812/archcid.66077 .

5. Zhan Y, Liu Z, Cheers C. Tumor necrosis factor alpha and interleukin-12 contribute to resistance to the intracellular bacterium Brucella abortus by different mechanisms. Infect Immun 1996; 64(7):2782-86.

6. Madej M, Nowak B, Świerkot J, Sokolik R, Chlebicki A, Korman L. et al. Cytokine profiles in axial spondyloarthritis. Reumatologia 2015; 53(1):9-13. doi: 10.5114/ reum.2015.50551.

7. Kobak S, Sever F, Ince O, Orman M. The prevalence of sacroiliitis and spondyloarthritis in patients with sarcoidosis. Int J Rheumatol 2014; 2014: 1-4. doi: $10.1155 / 2014 / 289454$.

8. Sveaas SH, Berg IJ, Provan SA, Semb AG, Olsen IC, Ueland T. et al. Circulating levels of inflammatory cytokines and cytokine receptors in patients with ankylosing spondylitis: a cross-sectional comparative study. Scand J Rheumatol 2015; 44(2):118-24. doi: 10.3109/03009742.2014.956142.

9. Landewé R, Braun J, Deodhar A, Dougados M, Maksymowych W, Mease P. et al. Efficacy of certolizumab pegol on signs and symptoms of axial spondyloarthritis including ankylosing spondylitis: 24-week results of a double-blind randomised placebo-controlled Phase 3 study. Ann Rheum Dis 2014; 73(1):39-47. doi: 10.1136/ annrheumdis-2013-204231.

10. van Royen BJ, Dijkmans BAC. Ankylosing Spondylitis: Diagnosis and Management. Taylor \& Francis; 2006.

11. Saadati N, Rajabian R. The effect of bisphosphonate on prevention of glucocorticoid-induced osteoporosis. Iran Red Cres Med J 2008; 10: 8-11.

12. Skendros P, Pappas G, Boura P. Cell-mediated immunity in human brucellosis. Microbes Infect 2011; 13(2):13442. doi: 10.1016/j.micinf.2010.10.015.

13. Saadi M, Karkhah A, Nouri HR. Development of a multi-epitope peptide vaccine inducing robust $\mathrm{T}$ cell responses against brucellosis using immunoinformatics based approaches. Infect Gen Evol 2017; 51:227-34. doi: 10.1016/j.meegid.2017.04.009.

14. Li J, Zhang C, Wang J-B, Chen S-S, Zhang T-P, Li S. et al. Relationship between the IL12B (rs3212227) gene polymorphism and susceptibility to multiple autoimmune diseases: a meta-analysis. Modern Rheumatol 2016; 26(5):749-56. doi: 10.3109/14397595.2016.1157282.

15. Skendros P, Boura P, Chrisagis D, Raptopoulou-Gigi M. Diminished percentage of CD4+ T-lymphocytes expressing interleukine-2 receptor alpha in chronic brucellosis. J Infecti 2007; 54(2):192-97. doi: 10.1016/j. jinf.2006.04.001.

16. Yin Y, Shi L, Chu Z, Jin W. A highly sensitive electrochemical IFN- $\gamma$ aptasensor based on a hierarchical graphene/ AuNPs electrode interface with a dual enzyme-assisted amplification strategy. RSC Adv 2017; 7(71):45053-60. doi: $10.1039 / \mathrm{c} 7 \mathrm{ra} 07817 \mathrm{j}$.

17. Rezazadeh M, Hajilooi M, Haidari M, Rafiei A, Ahmad Alavi S, Keramat F. Association of susceptibility to brucellosis and interleukin-4 promoter polymorphism. Scand J Infect Dis 2006; 38(11-12):1045-49. doi: 10.1080/00365540600786473.

18. Gaupp S, Cannella B, Raine CS. Amelioration of experimental autoimmune encephalomyelitis in IL-4R $\alpha-/-$ mice implicates compensatory up-regulation of Th2-type cytokines. Am J Pathol 2008; 173(1):119-29. doi: 10.2353/ ajpath.2008.071156.

19. Ghaznavi-Rad E, Khosravi K, Zarinfar N, Mosayebi G. Reduced IFN- $\gamma$ production in chronic brucellosis patients. Iran J Immunol 2017; 14(3):215-22.

20. Ganji A, Mosayebi G, Ghaznavi-Rad E. Evaluation of regulatory $\mathrm{T}$ cells in patients with acute and chronic brucellosis. Rep Biochem Mol Biol 2017; 5(2):91-96.

21. Akbulut H, Celik I, Akbulut A. Cytokine levels in patients with brucellosis and their relations with the treatment. Indian J Med Microbiol 2007; 25(4):387-90. doi: 10.4103/0255-0857.37345.

22. Hanan E-B, Saadany SE, Sahar E-Y, Amal E-B, Hassan A. Role of $\mathrm{T}$ helper-1 cytokine and nitric oxide production in patients with acute brucellosis. Tanta Med J 2005; 33:1088.

23. Campos PC, Gomes MT, Guimarães ES, Guimarães G, Oliveira SC. TLR7 and TLR3 sense Brucella abortus RNA to induce proinflammatory cytokine production but they are dispensable for host control of infection. Front Immunol 2017; 8:28. doi: 10.3389/fimmu.2017.00028

24. Najafipour S, Rasouli M, Kalani M, Seyed-Jalal-Aldin A-M, Moravej A. Association of interleukin-2 gene variants (positions +114 and- 384) and susceptibility to brucellosis in Iranian population. AIMS Mol Sci 2017; 4(1):103-09. doi: 10.3934/molsci.2017.1.103. 